\title{
STATE-VARIABLE MODELLING OF CLL RESONANT CONVERTERS
}

\author{
C. Gould, ${ }^{\dagger}$ D. A. Stone, M. P. Foster, \& C. Bingham \\ Department of Electronic and Electrical Engineering \\ University of Sheffield, Mappin Street, Sheffield, S1 3JD, UK \\ Tel. +44 (0) 1142225849 , Fax. +44 (0) 1142225196 \\ ${ }^{\dagger}$ Corresponding author: e-mail: d.a.stone@sheffield.ac.uk
}

Keywords: Harmonic excitation, network analysis, resonant converters, short-circuit protection, state-variable modelling.

\begin{abstract}
The paper presents the derivation and application of statevariable models to high-order topologies of resonant converters. In particular, a $3^{\text {rd }}$ order CLL resonant circuit is considered with bridge rectification and both a capacitive output filter (voltage output), and an LC output filter (current output). The state-variable model accuracy is verified against component-based simulation packages (Spice) and practical measurements, and it is shown that the resulting models facilitate rapid analysis compared to their integration-based counterparts (Spice, Saber), without the loss of accuracy normally associated with Fundamental Mode Approximation (FMA) techniques. Moreover, unlike FMA, the models correctly predict the resonant peaks associated with harmonic excitation of the tank resonance. Subsequently, it is shown that excitation of the resonant tank by odd harmonics of the input voltage can be utilised to provide overcurrent protection in the event of an output short-circuit. Further, through judicious control of operating frequency, it is shown that 'inductive' Zero Voltage Switching (ZVS) can still be obtained, facilitating reductions in gate-drive switching losses, thereby improving efficiency and thermal management of the supply under fault conditions. Although the results are ultimately generic to other converter counterparts, measured results from two prototype $36 \mathrm{~V}$ input, $11-14.4 \mathrm{~V}$ output, $3^{\text {rd }}$. order CLL converters are included to practically demonstrate the attributes of the proposed analysis and control schemes.
\end{abstract}

\section{Introduction}

Due to its low loss switching characteristics, the DC-DC resonant power converter topology allows excitation at much higher switching frequencies than traditional 'hard-switched' converters, enabling downsizing of reactive components. These lightweight, high power-density converters can be used in many diverse applications including military/aerospace (airborne radar/satellite), industrial power conversion, office automation (flat screen monitors/laptops), instrumentation (particularly medical), data communication, and even domestic appliances.

Traditionally, interest has been focused on 'second-order' resonant systems, based upon series or parallel topologies; however, classical analysis of the second-order converter neglects parasitic components that can be utilised in higherorder converter topologies.

These high-order resonant systems are termed 'series-parallel' and are often highly non-linear, complicating analysis, design and simulation. Despite this, they are desirable due to their ability to overcome the drawbacks experienced by more common second-order series or parallel converters. In particular, the $3^{\text {rd }}$ order series-parallel converter has been shown $[14,18-19]$ to operate with the advantages of series and parallel topologies whilst incorporating no-load regulation (which the series converter cannot achieve) and eliminating resonant circulating current independent of the load (a drawback of the parallel converter).

Of the many possible permutations of 3-element resonant topologies $[2,18-19]$, the CLL resonant topology (shown in Fig. 1 with half-bridge inverter, transformer, bridge rectifier and LC output filter) is of particular interest since the splitinductor arrangement can be used to take advantage of the leakage and magnetising inductances of an isolation transformer for high voltage applications. In some cases [12, 20], the magnetic circuit of the inductors and transformer can be integrated into one core, allowing higher efficiencies and greater overall power density. This use of the parasitic reactance is therefore highly beneficial in minimising volume envelopes.

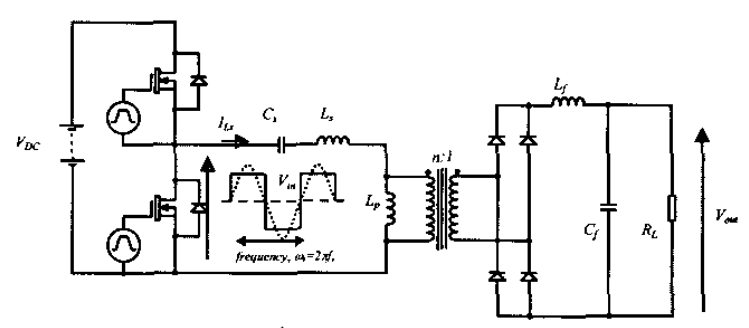

Fig. 1. CLL resonant converter topology (current output variant)

Aside from its desirability for use in volume-critical/high efficiency applications, the CLL resonant tank circuit also benefits from a load independent point (positioned at the series resonant frequency) that is higher than the resonant frequency [18]. This implies that supra-resonant switching will always impart inductive 'Zero Voltage Switching' (ZVS) characteristics from zero to full load (i.e. lagging power- 
factor), making the CLL converter an attractive choice for efficient power supplies.

Classically, the analysis and design of resonant converters is based on Fundamental Mode Approximation (FMA) [3, 8, 13, $15-16,19]$, or extensions and variants thereof $[6,7,9,11]$, where it is assumed that higher harmonics of the resonant currents and voltages are filtered out, enabling the interaction between the output filter, rectifier, and tank to be modelled by a simple 'equivalent resistor'. However, important harmonic characteristics of the converter are lost, producing inaccuracies in the technique when compared to practical results. Some authors have addressed such problems by augmenting the classical FMA 'equivalent resistor' model with additional components $[6,7,9,11]$ with some demonstrable success, at the expense of increasing the time required for the analysis. Others still have utilised Fourierseries analyses or frequency-domain approaches $[4,14,17]$ to provide more accurate results by applying $\mathrm{AC}$ analysis techniques to converters that incorporate all of the harmonics present in the system. Nevertheless, only steady-state frequency domain information is acquired. To obtain timedomain dynamic characteristics, integration-based simulation packages such as Spice or Saber must be used, incurring significant computational overheads. A need therefore remains for equation-based simulation models for resonant converters, and whilst LCC and LCLC variants have been previously considered $[1,10]$, the CLL counterpart (which is more complex to analyse) has yet to be addressed.

Here then, state-variable models for the voltage- and currentoutput variants of CLL converters are derived, and subsequently used for analysing their behaviour around the sub-harmonic resonant peaks - a feature not possible when using classical FMA. By comparison with previously reported fault protection mechanisms [21], it is shown that the proposed method affords improvements in efficiency and therefore converter thermal management.

\section{State-variable Modelling}

Each reactive component in Fig. 1 can be described by a state-variable that governs its action. For inductors, the voltage across an inductance is governed by the rate of change of current through it; similarly for capacitors, the current flowing through a capacitance is directly proportional to the rate of change of voltage across it. These rates of change become the state-variables, and can be combined using network theory to describe the behaviour of the converter. In order to achieve this, the converters are separated into fast and slow sub-systems related by a coupling equation. For these topologies, the fast sub-system represents the power switches and resonant circuit, whilst the slow system consists of the output filter. The coupling equation relating the two represents the highly non-linear behaviour of the rectifier.

Fig. 2 illustrates the use of an equivalent rectifier model comprising of on-state voltages, $V_{d}$, and resistances $r_{f}$ which combines the fast and slow sub-systems (shown in terms of their idealised reactive components and associated parasitic resistances). To avoid undue complication, the transformer shown in Fig. 1 is assumed to have a 1:1 turns ratio and is therefore modelled in Fig. 2 solely by its magnetising inductance, $L_{p}$, and winding resistance, $r_{L p}$. Likewise, a simple square input voltage and drain-source resistance, $r_{d s}$ models the MOSFET switches.

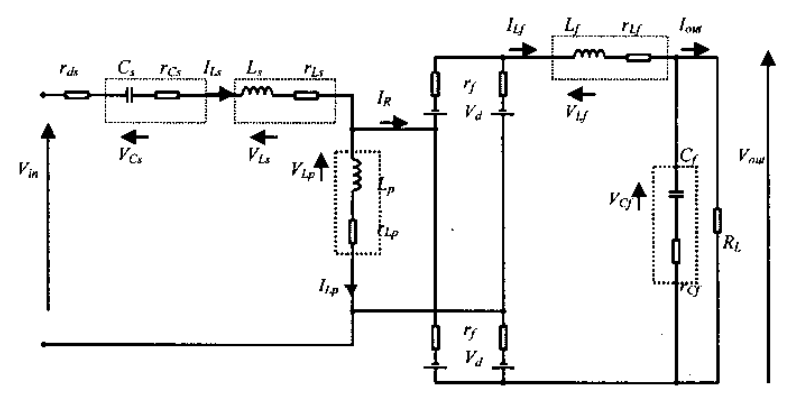

Fig. 2. State-variable diagram of CLL converter (current-output)

\subsection{Half-bridge CLL with bridge rectifier and voltage output}

In this case with the filter inductor $L_{f}$ and associated parasitic resistance $\left(r_{L_{f}}\right)$ in Fig. 2 being replaced by a short-circuit, the fast sub-system can be modelled by the following statevariable equations:

$$
\begin{gathered}
\frac{d I_{L_{s}}}{d t}=\frac{V_{\text {in }}-V_{C_{s}}-V_{L_{p}}-I_{L_{s}} \cdot r+I_{R} \cdot r_{L_{p}}}{L_{s}} \\
\frac{d I_{L_{p}}}{d t}=\frac{V_{L_{p}}}{L_{p}} \\
\frac{d V_{C_{s}}}{d t}=\frac{I_{L_{s}}}{C_{s}}
\end{gathered}
$$

where, $r=r_{d s}+r_{C_{s}}+r_{L_{s}}+r_{L_{p}}$. Similarly, the voltage across the output filter is given by:

$$
\begin{gathered}
\frac{d V_{C_{f}}}{d t}=\frac{\left|I_{R}\right| \cdot R_{L}}{C_{f} \cdot\left(R_{L}+r_{C_{f}}\right)}-\frac{V_{C_{f}}}{C_{f} \cdot\left(R_{L}+r_{C_{f}}\right)} \\
V_{\text {out }}=V_{C_{f}}+\frac{d V_{C_{f}}}{d t} \cdot C_{f} \cdot r_{C_{f}}
\end{gathered}
$$

Considering the voltages across the input and output of the rectifier, a coupling equation governing the action of the rectifier can be derived.

$$
V_{L_{p}}=\operatorname{sgn}\left(I_{R}\right) \cdot\left[V_{\text {out }}+2 V_{d}+2\left|I_{R}\right| \cdot \dot{r}_{f}\right]-I_{L_{p}} \cdot r_{L_{p}}
$$

where $V_{d}$ is diode voltage drop, and from network theory:

$$
I_{L_{p}}=I_{L_{s}}-I_{R}
$$




\subsection{Model Verification}

A prototype CLL voltage-output converter has been designed using an FMA procedure [13] to provide a 11-14.4V output from a $36 \mathrm{~V}$ input supply, resulting in the following (measured) component values: $r_{d s}=0.19 \Omega, C_{s}=23 n F$, $r_{C_{s}}=46 \mathrm{~m} \Omega, L_{s}=54.2 \mu \mathrm{H}, r_{L_{s}}=0.7 \Omega, L_{p}=29.9 \mu \mathrm{H}$, $r_{L_{p}}=0.7 \Omega, \quad V_{d}=0.8 V, \quad r_{f}=1 \Omega, \quad C_{f}=100 \mu \mathrm{F}$, $r_{C_{f}}=0.44 \Omega, R_{L}=20 \Omega$. The resonant frequency is $f_{r}=128 \mathrm{kHz}$.

Time-domain simulation of the state-variable model for operation at $142.7 \mathrm{kHz}$ produces the waveforms shown in Figs. 3(a) \& 4(a). For comparison, the same converter has been simulated in Spice, producing the waveforms shown in Figs. 3(b) \& 4(b). Evidently the state-variable model provides comparable accuracy to Spice, however it is of note that the state-variable model requires less than $1 / 10^{\text {th }}$ of the execution time.

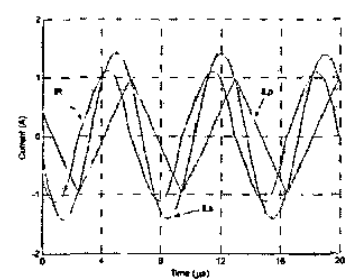

(a)

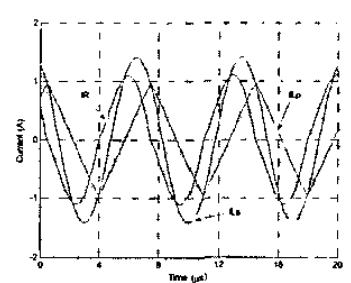

(b)
Fig. 3. Currents of the voltage-output CLL converter (a) State-variable results (b) results from Spice

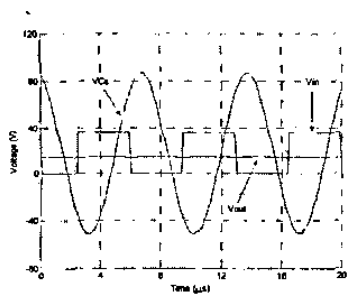

(a)

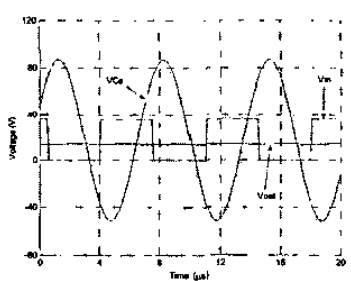

(b)
Fig. 4. Voltages of the voltage-output CLL converter (a) State-variable results (b) results from Spice

\subsection{Half-bridge CLL with bridge rectifier and current output}

With the inclusion of $L_{f}$ and $r_{L_{f}}$, the fast sub-system model remains identical to that of the voltage-output converter and can therefore be described using equations (1-3). The slow sub-system now contains an inductor hence requires an additional state-variable:

$$
\frac{d I_{L_{f}}}{d t}=\frac{V_{L_{f}}}{L_{f}}
$$

Whilst equations (5) and (7) still hold, the inductor $L_{f}$ now governs the current into the output filter, hence equation (4) is modified:

$$
\frac{d V_{C_{f}}}{d t}=\frac{I_{L_{f}} \cdot R_{L}}{C_{f} \cdot\left(R_{L}+r_{C_{f}}\right)}-\frac{V_{C_{f}}}{C_{f} \cdot\left(R_{L}+r_{C_{f}}\right)}
$$

However, for this converter topology the filter inductor also imposes an additional mode of operation on the rectifier. This mode is active when the filter inductor limits the rate of change of current drawn by the output filter, beginning a period where $I_{L_{f}}>\left|I_{R}\right|$ and all four diodes are conducting

(Fig. 5).

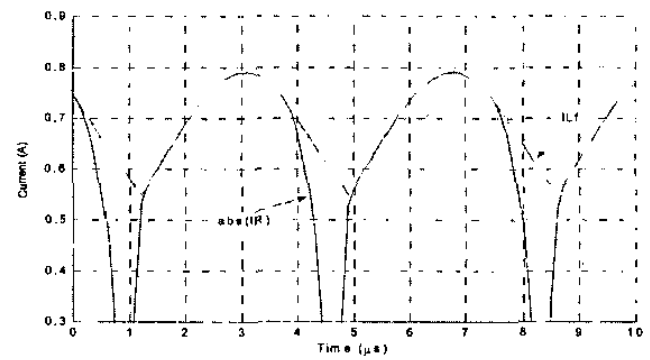

Fig. 5. Rectifier short-circuit action

During this period the rectifier effectively becomes a shortcircuit. In this condition the resonant circuit and filter circuit operate independently, complicating the state-variable model, making it necessary to switch between two modes of operation:

i) Conduction mode: $\left|V_{L_{p}}\right|=0$ hence $I_{L_{f}}=\left|I_{R}\right|$, and the converter behaves similarly to the voltage-output variant.

ii) Short-circuit mode: $\left|V_{L_{p}}\right|=0$ hence $I_{L_{f}}>\left|I_{R}\right|$, and the fast and slow sub-systems are independent, and the converter can be described by two circuits shown in Fig. 6:

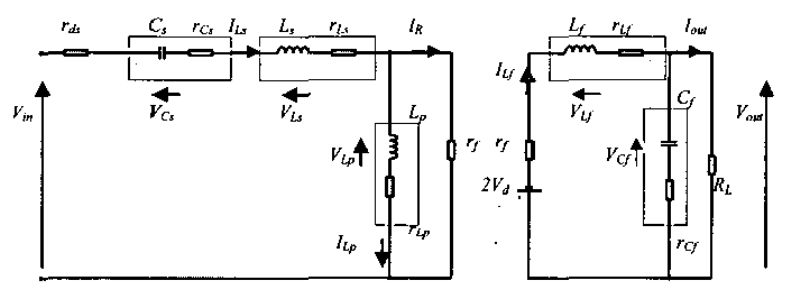

Fig. 6. Equivalent circuit of converter operating in short-circuit mode

In the conduction mode the rectifier operates as a normal bridge, hence $I_{L_{f}}$ is given by the magnitude of the rectifier current, $\left|I_{R}\right|$ : 


$$
\frac{d I_{L_{f}}}{d t}=\frac{d\left|I_{R}\right|}{d t}=\operatorname{sgn}\left(I_{R}\right) \cdot \frac{d I_{R}}{d t}
$$

In a similar manner as before, equating voltages across both sides of the rectifier produces the coupling equation:

$$
\left.V_{L_{p}}=\operatorname{sgn}\left(I_{R}\right) \cdot\left|V_{\text {out }}+2 V_{d}+V_{L_{f}}+I_{L_{f}} \cdot r_{L_{f}}+2\right| I_{R} \mid \cdot r_{f}\right]-I_{L_{p}} \cdot r_{L_{p}}
$$

In the short-circuit condition (as shown by the equivalent circuit in Fig. 6) state-variable analysis yields:

$$
\frac{d I_{L_{f}}}{d t}=-\frac{1}{L_{f}}\left[V_{\text {out }}+2 V_{d}+I_{L_{f}} \cdot\left(r_{f}+r_{L_{f}}\right)\right]
$$

and since there is no coupling between the two systems, $V_{L_{p}}$ is now determined from:

$$
V_{L_{p}}=I_{R} \cdot r_{f}-I_{L_{p}} \cdot r_{L_{p}}
$$

To produce accurate results, switching between each circuit needs to occur at the correct times. The short-circuit mode can only occur when $I_{L_{f}}>\left|I_{R}\right|$, which, in turn, only occurs when $\left|V_{L_{p}}\right|=$. Thus, the short-circuit mode is entered as $\left|V_{L_{p}}\right|$ reaches zero. However, when in this mode, $V_{L_{p}}$ will always be equal to zero volts, and the boundary time for switching back to the conduction mode has to be taken at the instant when $I_{L_{f}}=\left|I_{R}\right|$ again.

\subsection{Model Verification}

The prototype converter previously described is now augmented with an output filter inductor with $L_{f}=100 \mu \mathrm{H}$, $r_{L_{f}}=0.7 \Omega$, giving the resonant frequency of the currentoutput counterpart as $f_{r}=122 \mathrm{kHz}$. Operation at $135 \mathrm{kHz}$ is examined to provide equivalent output voltages to the voltage-output converter and simulated waveforms at this frequency are shown in Figs. $7 \& 8$ for both the state-variable model and Spice. Once again, comparable accuracy in the time-domain is shown, however, of note in this case, is that due to the additional complexity required for describing the additional operating modes, only marginally faster execution speeds are achieved by state-variable over Spice methods. Nevertheless, a key advantage of using the state-variable model over Spice exists since it provides a convenient mechanism for the structured design of a feedback controller; a feature not supported by component-based simulation techniques.

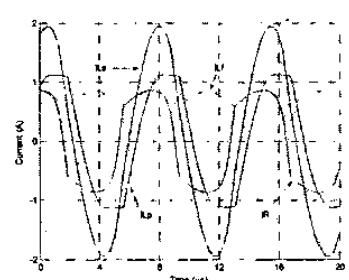

(a)

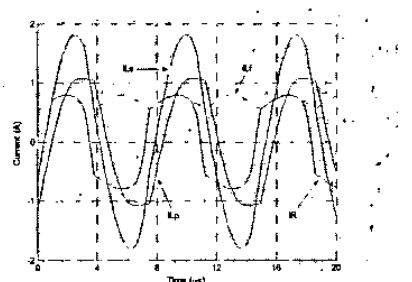

(b)
Fig. 7. Currents of the current-output CLL converter (a) State-variable results (b) results from Spice

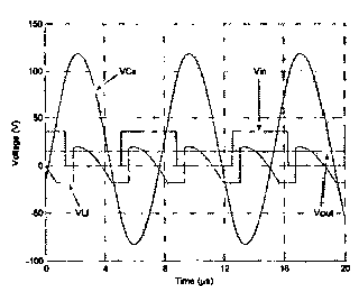

(a)

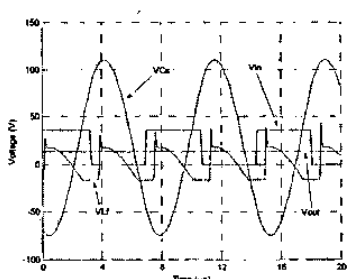

(b)
Fig. 8. Voltages of the current-output CLL converter (a) State-variable results (b) results from Spice

Since resonant converters are often employed with frequencybased self-regulation techniques, frequency-domain. simulations must provide the same degree of accuracy as the time-domain solutions. In this case, the state-variable models are compared against results obtained from the prototype converters and traditional FMA predictions to show the improvement in performance. These results are shown in Fig. 9 , where it can be seen that the frequency domain characteristics of the practical converters are indeed captured by the state-variable models.

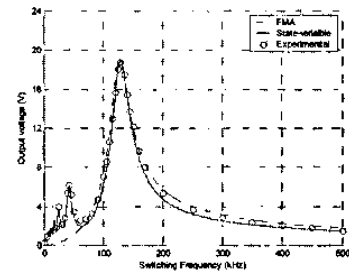

(a)

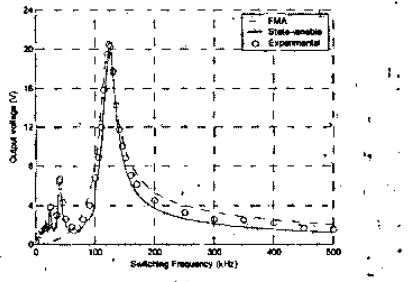

(b)
Fig. 9. Output voltage as a function of excitation frequency from the statevariable models, FMA, and measurements from the prototype converters: (a) voltage-output topology (b) current-output topology

\section{Sub-harmonic control of a resonant converter}

At relatively low operating frequencies both converters exhibit multiple resonant peaks when compared to the FMA predictions. These peaks arise from harmonics inherent in the square input voltage applied across the resonant tank. However, the FMA prediction assumes that the resonant tank filters out these harmonics. Considering a Fourier representation of the input voltage [5], excitation of the resonant tank at a third of the system's resonant frequency will simultaneously excite the resonant frequency via the third 
harmonic, albeit at a third of the magnitude (similarly with the fifth, seventh, etc).

By way of example, the current-output converter above has been utilised to provide $36-3.3 \mathrm{~V} \mathrm{DC-DC}$ conversion, as may be required in an electric vehicle application, for instance. Fig. 10 demonstrates that equivalent output voltages may be obtained through operation around these sub-harmonics of the resonant frequency.

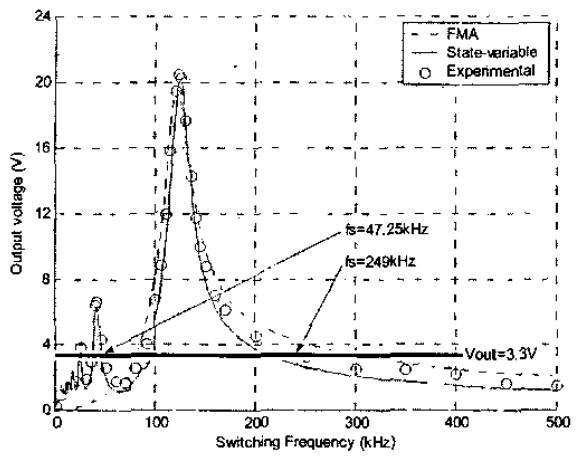

Fig. 10. Proposed operating points

For this example the desired $3.3 \mathrm{~V}$ output may be obtained classically through supra-resonant ZVS (i.e. inductively switched at $249 \mathrm{kHz}$ as per Fig. 11 (a)), or by switching close to the third harmonic at $47.25 \mathrm{kHz}$. Moreover, if the converter is excited on the 'inductive' side of the third harmonic, the series inductor current lags the excitation voltage and ZVS is retained (Fig. 11 (b)).

It is also apparent from Fig. 11 (b) that although the resonant tank is being excited at $47.25 \mathrm{kHz}$, due to the harmonic action at that frequency the tank responds primarily to 3 multiples of $47.25 \mathrm{kHz}$, i.e. $141.75 \mathrm{kHz}$ to develop the output voltage. In Fig. 10 the expected output voltage at $141.75 \mathrm{kHz}$ is approximately $10 \mathrm{~V}$ and demonstrates the attenuation near the third harmonic to one third of $10 \mathrm{~V}(3.3 \mathrm{~V})$.

Comparing this method of tank excitation to classic supraresonant switching highlights the reduction in necessary switching frequency (by 5 times in this case). Hence there is a subsequent reduction in gate-drive power requirements and high-frequency radiated/conducted EMI by virtue of loading the supply $\left(V_{D C}\right)$ with a lower fundamental frequency.

For this particular case, the measured gate-drive power dissipation reduces from $0.8 \mathrm{~W}$ at $249 \mathrm{kHz}$ to $0.2 \mathrm{~W}$ at $47.25 \mathrm{kHz}$

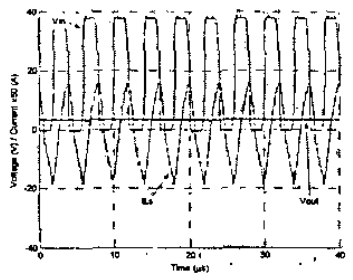

(a)

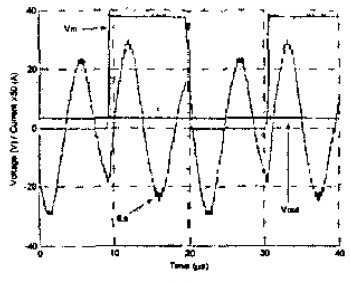

(b)
Fig. 11. Measured waveforms of current-output converter excited at (a) $249 \mathrm{kHz}$ and (b) $47.25 \mathrm{kHz}$

\section{Over-current Protection Through Harmonic Excitation}

Methods for fault protection of the LCL series resonant converter (effectively a CLL resonant tank) have been recently reported [21] that deal with an output short-circuit scenario. During an output short-circuit, the electrical stresses on the components increase significantly, and can often exceed those experienced at resonance under full-load conditions (i.e. the maximum rated stresses). Naturally, under these conditions the converter is susceptible to critical damage. By way of example, Fig. 12 shows the currents in the voltage-output converter when operated at $150 \mathrm{kHz}$ for (a) normal operation i.e. full-load $(20 \Omega)$ and in the case of a short circuit (b) when the load tends to zero. In this case the electrical stresses can be seen to increase by a multiple of three, which are above the rated stresses at resonance.

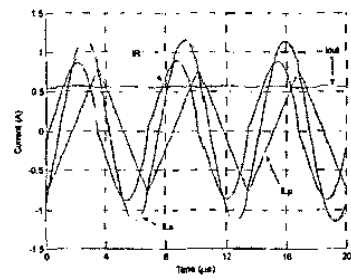

(a)

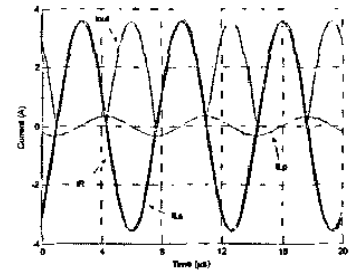

(b)
Fig. 12. Converter currents during operation at $150 \mathrm{kHz}$ (a) normal operation and (b) during an output short-circuit

Among the fault protection methods suggested [21], the simplest by far is to significantly increase the excitation frequency (to retain ZVS) until the stresses fall within acceptable limits. For the voltage-output converter above, Fig. 13(a) shows the result of increasing the switching frequency to $183 \mathrm{kHz}$ after the converter output is short-circuited, whereupon the tank- and output-currents can be seen to remain within normal specifications. However, although the currents are limited, a proportional increase in gate-drive switching losses ultimately results. For converters with low differential gain (as a function of frequency), this increase in frequency may have to be particularly large (say, up to $400 \mathrm{kHz}$ ), incurring significantly greater losses in the MOSFETs.

Alternatively however, ZVS can also be obtained by moving down in frequency to excite the tank with the $3^{\text {rd }}$ harmonic of the input voltage. In particular, since the parallel branch of the tank is effectively short-circuit during the fault, the converter behaves similarly to the LC series resonant converter and hence achieves maximum gain at the series resonant frequency. It is possible then to reduce the excessive stresses experienced at this load by reducing the switching frequency to $\approx 1 / 3^{\text {rd }}$ of the series resonant frequency, and exciting the resonant tank using the $3^{\text {rd }}$ harmonic of the input excitation voltage - see Fig. 9(a).

This scheme has the advantage of reducing gate-drive losses, thereby improving efficiency, and importantly the thermal management of the supply. By way of example, the circuit currents when operating at a switching frequency of $51 \mathrm{kHz}$ 
during an output short-circuit, are shown in Fig. 13(b), and can be seen to stay within normal design limits. More importantly, by actually reducing the excitation frequency, the losses attributed to the gate-drive circuitry decrease, thereby improving efficiency and reducing thermal output. Specifically, for operation depicted in Fig. 13(a), the gatedrive losses with the converter operating at $183 \mathrm{kHz}$ were measured at $0.6 \mathrm{~W}$, whilst at $51 \mathrm{kHz}$ (Fig. 13(b)) they were just $0.2 \mathrm{~W}$ - an overall reduction of $\approx 66 \%$.

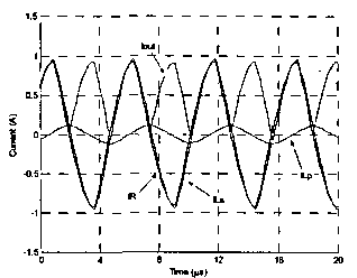

(a)

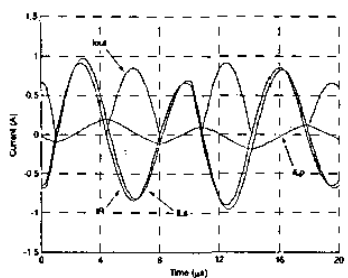

(b)
Fig. 13. Circuit currents when the output is short-circuit (a) operation at high excitation frequency (b) operation with low excitation frequency in the region of an odd sub-multiple $(1 / 3 \mathrm{rd})$ of the series resonant frequency.

Finally, whilst the study has considered the $3^{\text {rd }}$ order CLL voltage-output converter to demonstrate the short-circuit fault control scheme, the technique is ultimately applicable to other common high-order converter topologies, e.g. LCLC and LCC variants.

\section{Conclusions}

State-variable models of a 3rd order CLL resonant converter are developed for both current- and voltage-output applications. Their accuracy is proven to be commensurate with SPICE models and has a similar order, or faster, execution time. The inclusion of an inductor in the output filter network is shown to complicate circuit operation, analysis and simulation of the current-output converter. Prototype converters are commissioned as a proof of analysis. The experimental results demonstrate the improved accuracy of the state-variable models over traditional FMA predictions, through modelling of the 'true' square wave input to the resonant tank.

Operation of resonant dc-dc converters around odd submultiples of the tank resonant frequency is proposed to facilitate reduced switching losses and reduced EMI. It is shown that allowing the resonant tank to operate on harmonics of the excitation voltage i.e. at reduced operating frequencies, allows output voltages commensurate with those obtained by supra-resonant switching. Moreover, converter operation around the odd sub-multiples of the tank resonant frequency has been proposed to limit the output fault current and component stresses in the event of a short-circuit. By comparison with previously published methods, practical measurements have demonstrated that significant loss reductions can be attributed to the proposed technique whilst maintaining low switching losses by preserving inductive switching (ZVS) of the power devices.

\section{References}

[1] Ang, Y., Foster, M.P., Sewell, H.l, Bingham, C.M., Stone, D.A. 'Stress analysis of fourth-order LLCC resonant converters', IEE Electronics Letters, Vol: 38 (24), ppl585-1586, Nov. 2002.

[2] Batarseh, I., 'Resonant Converter Topologies with Three and Four Energy Storage Elements', IEEE Trans. Power Electronics, vol. 9 no. 1, pp 64-73, Jan. 1994

[3] Bhat, A.K.S., Dewan, S.B., 'Analysis and design of a high-frequency resonant converter using LCC-type commutation'. IEEE Transactions ' on Power Electronics. Vol. pe-2, no.4 pp 291-300 October 1987.

[4] Bhat, A.K.S., Dewan, S.B., 'A generalized approach for the steady-state analysis of resonant inverters'. IEEE Trans. on Industry Applications, Vol. 25, no.2 pp 326-338 March/April 1989.

[5] Erickson, R. W., 'Fundamentals of Power electronics', Chapman \& Hall, pp717-755, 1997.

[6] Forsyth, A. J., Ward, G. A., Mollov, S. V., 'Extended Fundamental Frequency Analysis of the LCC Resonant Converter', IEEE Trans. On Power Electronics, Vol: 18 (6), pp1286-1292, Nov. 2003.

[7] Forsyth, A. J., Mollov, S.V., 'Simple equivalent circuit for the seriesloaded resonant converter with voltage boosting capacitor,', IEE Proc. Electric Power Applications, Vol. 145, (4), p.301-306, July 1998.

[8] Foster, M. P., Sewell, H. I., Bingham, C. M., Stone, D. A., Hente, D., Howe, D., 'High Speed Analysis of Resonant Power Converters', IEE Proc. Electric Power Applications, 150 (1), pp.62-70, 2003.

[9] Sewell, H.I., Foster, M. P., Bingham, C. M., Stone, D. A., Hente, D. Howe, D., 'Analysis of voltage output LCC resonant converters, including boost mode operation', IEE Proceedings On Electric Power Applications, Vol: 150 (6), pp629-756, November 2003.

[10] Foster, M. P., Sewell, H. I., Bingham, C. M., Stone, D. A., 'Statevariable Modeling of LCC Voltage Output Converters', IEE Electronics Letters, (17), pp.1065-1066, 2001.

[11] Hayes, J.G., Egan, M.G., 'Rectifier-compensated Fundaments Mode Approximation Analysis of the series-parallel LCLC family of resonant converters with capacitive filter and voltage-source load' PESC'99 record 30th IEEE Power Electronics Specialist Conference, vol.2 pp1030-1036, 1999.

[12] Kats, A., Ivensky, G., Ben-Yaakov, 'Application of Integrated Magnetic in Resonant Converters', Twelfth Annual IEEE Applied Power Electronics Conference and Exposition (APEC) 1997, vol. 2, pp 925-930, 1997

[13] Kazimierczuk, M. K., Czarkowski, D., 'Resonant Power Converters', Wiley, pp 437-448, April 1995.

[14] Lazar, J. F., Martinelli, R., 'Steady-state Analysis of the LLC Series Resonant Converter', Sixteenth Annual Applicd Power Electronics Confercnce and Exposition. Conference Proceedings, p728 735 vol2, 2001.

[15] Mandhana, O. P., 'Computer aided analysis and design of load commutated resonant converters', PESC 96 Record. 27th Annual IEEE Power Electronics Specialists Conference. vol.1. pp279-85 1996.

[16] Raju, G. S. N., Doradla,-S., "An LCL resonant converter with PWM control-analysis, simulation, and implementation', IEEE Transactions on Power Electronics. Vol: 10 (2), pp 164-74. March 1995.

[17] Raju, G. S. N., Doradla,-S., 'A novel LCL resonant converter with PWM control - analysis, simulation, and implementation', IEEE Appl. Power Electr. Conf. Rec., pp 998-1004, 1994.

[18] Severns, R.P., 'Topologies for three element resonant converters', IEEE Trans. Power Electronics, vol. 7, no. 1, pp 89-98, Jan. 1992.

[19] Steigerwald, R. L., 'A Comparison of Half-Bridge Resonant Converter Topologies', IEEE Trans. Power Electronics, vol, 3, no. 2, pp174-182, April 1988.

[20] Yang, B., Chen, R., Lee, F.C., 'Integrated Magnetic for LLC Resonant Converter', Seventeenth Annual IEEE Applied Power Electronics Conference and Exposition (APEC) 2002, pp 346-351, 2002

[21] Yang, B., Lee, F.C., Concannon, M., 'Over Current Protection Methods for LLC Resonant Converter' Eighteenth Annual IEEE Applied Power Electronics Conference and Exposition (Proceedings), p605-609, 2003. 